\title{
Clinical experience with antifibrotics in fibrotic hypersensitivity pneumonitis: a 3-year real-life observational study
}

\author{
Vasilios Tzilas ${ }^{1,4}$, Argyris Tzouvelekis ${ }^{1,4}$, Evangelos Bouros ${ }^{1}$, \\ Theodoros Karampitsakos (1) 1, Maria Ntassiou', Eleni Avdoula1, \\ Athena Trachalaki (1) ${ }^{2}$, Katerina Antoniou², Ganesh Raghu ${ }^{3}$ and \\ Demosthenes Bouros (iD) ${ }^{1}$
}

Affiliations: ${ }^{1}$ First Academic Dept of Pneumonology, Interstitial Lung Diseases Unit, Hospital for Diseases of the Chest, "Sotiria", Medical School, National and Kapodistrian University of Athens, Athens, Greece. ${ }^{2}$ Dept of Pneumonology, Medical School, University of Crete, Crete, Greece. ${ }^{3}$ Division of Pulmonary, Sleep and Critical Care Medicine, University of Washington, Seattle, WA, USA. ${ }^{4}$ These authors contributed equally.

Correspondence: Demosthenes Bouros, Hospital for Chest Diseases "Sotiria”, Messogion 152, Athens 11527, Greece. E- mail: dbourosamed.uoa.gr

\section{ABSTRACT}

Background: Fibrotic hypersensitivity pneumonitis (f-HP) can exhibit a progressive course similar to idiopathic pulmonary fibrosis (IPF). The lack of diagnostic guidelines and randomised controlled trials in this population represent a significant unmet need.

Objectives: To describe our clinical experience with antifibrotics in patients with f-HP.

Material and methods: Retrospective study of 30 patients diagnosed with f-HP upon re-evaluation within a multidisciplinary team discussion of 295 consecutive patients (January 2012 to December 2017) who had been diagnosed initially with IPF at outside facilities and were referred to our centres.

Results: Pirfenidone was initially administered to $14(46.7 \%)$ patients and nintedanib to 16 (53.3\%) patients. There were $26(86.7 \%)$ males, with mean \pm SD age $70.2 \pm 8.4$ years. The annual rate of decline in forced vital capacity (FVC) \% predicted over the 3-year treatment period adjusted for baseline FVC \% pred measurement was $4.2 \%$ (95\% CI $1.9-6.6 \%, \mathrm{p}=0.001)$ and $7.5 \%$ (95\% CI $3.3-11.7 \%$; $\mathrm{p}=0.001$ ) in imputation analysis. The annual rate of decline in diffusing capacity of the lung for carbon monoxide ( $\left.D_{\text {LCO }}\right) \%$ predicted throughout the 3-year treatment period adjusted for baseline $D_{\text {LCO }} \%$ pred was $5.7 \%$ (95\% CI 3.1-8.4\%, p<0.001) and 5.8\% (95\% CI 3.4-8.1\%, $\mathrm{p}<0.001)$ in imputation analysis. The nature of adverse events was related to the type of antifibrotic agent administered.

Conclusion: In patients with $\mathrm{f}-\mathrm{HP}$ receiving antifibrotics there is a statistically significant annual decline in FVC $\%$ pred and $D_{\text {LCO }} \%$ pred over a period of 3 years. Prospective randomised trials exceeding 1 year are warranted to determine the long-term efficacy of antifibrotics.

@ERSpublications

In patients with fibrotic hypersensitivity pneumonitis receiving antifibrotics, there is a statistically significant annual decline in FVC \% predicted and $D_{\mathrm{LCO}} \%$ predicted over a period of 3 years. Prospective randomised trials exceeding 1 year are needed. https://bit.ly/3abhAL9

Cite this article as: Tzilas V, Tzouvelekis A, Bouros E, et al. Clinical experience with antifibrotics in fibrotic hypersensitivity pneumonitis: a 3-year real-life observational study. ERJ Open Res 2020; 6: 00152-2020 [https://doi.org/10.1183/23120541.00152-2020].

This article has been revised according to the erratum published in ERJ Open Res 2021; 7: 50152-2020 [https://doi.org/ $10.1183 / 23120541.50152-2020]$.

Received: 28 March 2020 | Accepted after revision: 3 Aug 2020 | First published: 2 Nov 2020

Copyright $\odot$ ERS 2020. This article is open access and distributed under the terms of the Creative Commons Attribution Non-Commercial Licence 4.0. 


\section{Introduction}

Currently, there are no guidelines for diagnosis and management of fibrotic hypersensitivity pneumonitis (f-HP), and thus the management of patients with f-HP is deferred to the clinician's best judgment. Even among experienced multidisciplinary teams, agreement levels on diagnosis are variable [1]. HP pathogenesis is considered to be based on an abnormal exaggerated immune response to persistent inhaled antigens [2]. Therefore, current treatment consists of corticosteroids and immunosuppressants in addition to avoidance of further antigen exposure, if identified. Other than recent perspectives, there are no clinical practice guidelines to date for ascertaining the diagnosis or management of HP [3-7].

The presence of histopathological and/or radiological fibrosis in the context of HP has been robustly correlated to poor survival in several studies [8-10]. Certain phenotypes of radiological fibrosis, such as the presence of honeycombing, seem to correlate with even worse survival [11]. Indeed, despite antigen avoidance and administration of immunosuppressants, some patients with f-HP exhibit a continuous deteriorating course, resembling the relentless course of idiopathic pulmonary fibrosis (IPF). These patients present a clear unmet need. IPF and f-HP seem to share common pathogenetic mechanisms $[12,13]$. Thus, the use of antifibrotics in $\mathrm{f}-\mathrm{HP}$ appears to be a promising option, especially in light of a recent study showing beneficial effects in patients with non-IPF progressive fibrotic interstitial lung diseases (ILDs) [14].

The diagnosis of ILDs is a dynamic process requiring close interaction and discussion between pulmonologist, radiologist and pathologist [15]. The change of diagnosis in light of new data is a well-observed phenomenon in ILDs. A characteristic example is a change of diagnosis from IPF to HP [16-18]. In this context we identified 30 patients with f-HP treated only with antifibrotics from a cohort of 295 patients with an initial diagnosis of IPF from the primary physicians, who were referred to our centres for a second expert opinion. We aimed to describe our clinical experience with antifibrotics in these patients with f-HP.

\section{Materials and methods}

Patients with f-HP receiving antifibrotics were identified retrospectively from a cohort of consecutive patients referred to the ILD departments of the 1st Respiratory University Clinic of the National and Kapodistrian University of Athens at the Chest Diseases Hospital Sotiria (Athens, Greece), and to the ILD department of the Respiratory University Clinic of the University of Crete (Heraklion, Greece) between January 2012 and December 2017. The study was approved by the institutional review board (27249-20/ $11 / 19)$. Due to the retrospective nature of the study, no informed consent was required. From a total of 295 consecutive patients who had been diagnosed initially with IPF per 2011 criteria [19] at outside facilities and were treated with antifibrotics, we identified 38 patients with a final diagnosis of f-HP. Management solely with antifibrotics continued for 30 patients, while eight received a combination of antifibrotics and immunosuppression and were excluded from the study (figure 1). Dosing for pirfenidone was $801 \mathrm{mg}$ three times daily and for nintedanib $150 \mathrm{mg}$ twice daily. Diagnosis of $\mathrm{f}-\mathrm{HP}$ was established in the context of multidisciplinary team discussion and further evaluation including bronchoalveolar lavage (BAL) lymphocytosis, surgical lung biopsy (SLB) and high-resolution computed tomography (HRCT). It was based on the presence of at least two of the following criteria: identified exposure to a compatible inciting antigen, presence of mosaic attenuation on HRCT, histological findings supporting the diagnosis of HP (presence of giant cells, noncaseating granulomas, bridging fibrosis, airway-centred fibrosis) [2-4] In all cases, where an inciting antigen was identified, successful interventions aiming at antigen removal were verified. The presence of BAL lymphocytosis $>20 \%$ was used as supportive of f-HP diagnosis in cases with inconsistent with a usual interstitial pneumonia (UIP) pattern due to the presence of mosaic attenuation [18]. The diagnosis of f-HP was established locally in each centre after discussion and integration of the clinical, radiological and pathological findings in a multidisciplinary conference. Pulmonary hypertension was defined as right-ventricle systolic pressure $>35 \mathrm{mmHg}-$ central venous pressure measured by echocardiography.

\section{Data analyses and statistical methods}

The normality of distribution of continuous variables was examined using the Shapiro-Wilk test. Continuous variables are presented as mean $\pm S D$ if data followed a normal distribution, or median (interquartile range (IQR)) if not. Linear mixed models with forced vital capacity (FVC) \% predicted values as the dependent variable, and time (continuous) and baseline FVC \% pred as independent variables, using random intercept and slope with a variety of covariance structures, were fitted to estimate the annual rate of decline in FVC \% pred adjusted for baseline measurement. Information criteria (Akaike information criterion, Akaike information criterion corrected and Bayesian information criterion) were used in order to select the appropriate covariance structure of the models. The baseline-adjusted estimates of FVC \% pred at 1, 2 and 3 years are presented along with $95 \%$ confidence intervals. Similar analysis was 


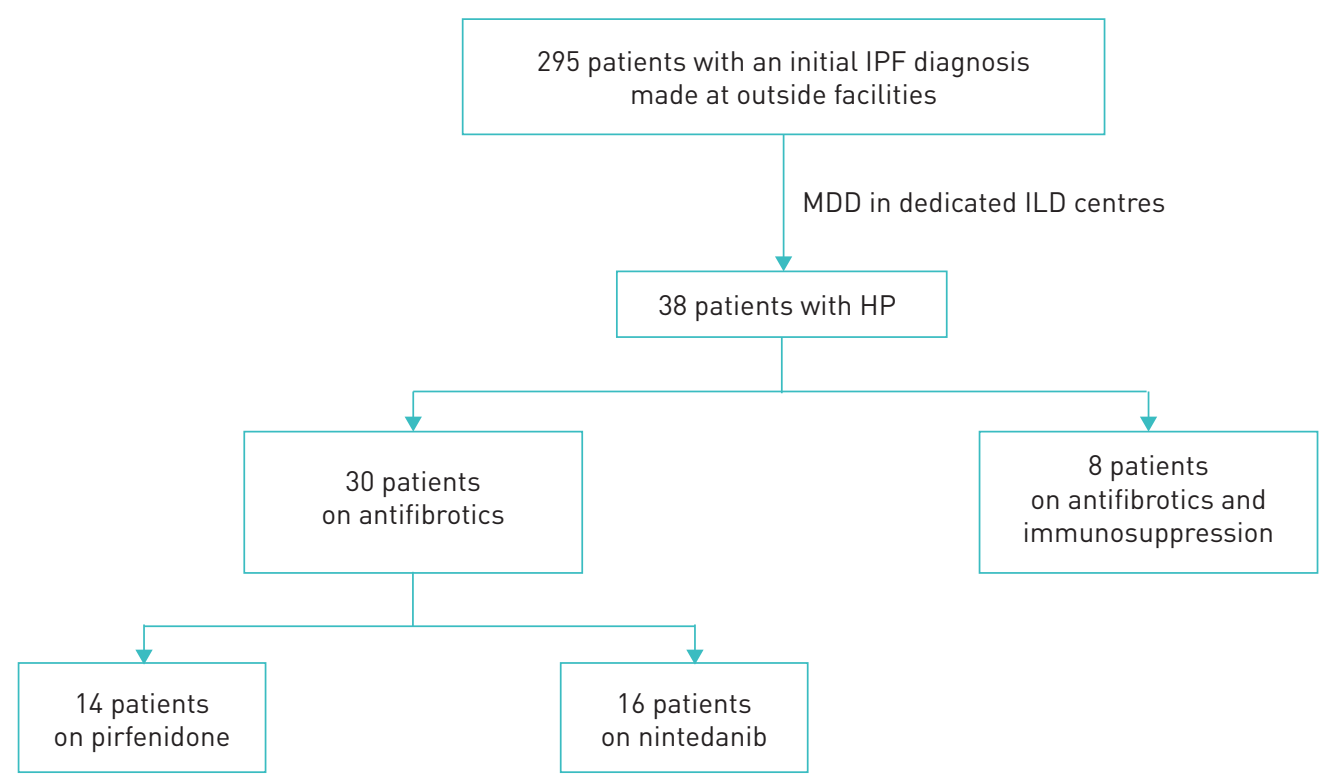

FIGURE 1 Flowchart of the study. Multidisciplinary team discussion (MDD) was performed by two pulmonologists experienced in interstitial lung disease (ILDs), a thoracic radiologist and a pathologist in cases with available lung biopsy. There was one patient with a usual interstitial pneumonia (UIP) pattern land bronchoalveolar lavage (BAL) lymphocytosis $>20 \%$ ), five patients with possible UIP pattern ( $n=3$ with BAL lymphocytosis $>20 \%$ ) and 24 patients with mosaic attenuation, most $(n=16)$ with "head and cheese sign" ( $n=6$ with BAL lymphocytosis > 20\%). IPF: idiopathic pulmonary fibrosis; HP: hypersensitivity pneumonitis.

conducted for the estimation of the baseline-adjusted annual rate of decline in $D_{\text {LCO }} \%$ pred over the 3 -year treatment period.

In addition, the aforementioned analyses were conducted using imputation for data missing owing to death. The missing values of FVC $\%$ pred due to death were imputed with $30 \%$, while the respective $D_{\text {LCO }}$ $\%$ pred values were imputed with 15\%. At 2 and 3 years post-baseline, two and four patients had died, respectively, and their FVC and $D_{\mathrm{LCO}}$ measurements were imputed. All other missing data were not imputed.

The statistical analysis was performed using the software package SAS (v9.4; SAS Institute, Cary, NC, USA); $<<0.05$ was considered statistically significant.

\section{Results}

Demographic characteristics

Among the 30 patients with $\mathrm{f}-\mathrm{HP}$ receiving antifibrotics, the majority were male (86.4\%) with mean $\pm \mathrm{SD}$ age $70.2 \pm 8.4$ years; $63.3 \%$ were ex-smokers. The median (IQR) antifibrotic treatment duration over the 3 -year observation period was $24.0(12.0-36.0)$ months. The median (IQR) overall duration of exposure to nintedanib $(\mathrm{n}=19)$ and pirfenidone $(\mathrm{n}=15)$ was $24.0(12.0-36.0)$ months for each (including the exposure to the respective treatment after switching from the one agent to the other among the switchers). The median (IQR) time from treatment initiation until discontinuation/switch among patients initiated on nintedanib $(\mathrm{n}=16)$ and those initiated on pirfenidone $(\mathrm{n}=14)$ was $18.0(12.0-36.0)$ and $19.0(12.0-36.0)$ months, respectively.

Baseline mean \pm SD FVC was $74.2 \pm 18.3 \%$ pred and $D_{\text {LCO }}$ was $46.9 \pm 16 \%$ pred. Initially, 14 (46.7\%) patients received pirfenidone and $16(53.3 \%)$ patients received nintedanib. An inciting antigen was identified in 25 (83.3\%) patients. SLB was performed in seven $(23.3 \%)$ patients. The most commonly reported comorbidities were arterial hypertension and gastro-oesophageal reflux disease, which were present in 13 (43.3\%) and $12(40 \%)$ patients, respectively. Coronary artery disease was present in six (20\%) patients. Pulmonary hypertension was identified in nine $(30 \%)$ patients based on transthoracic echocardiography. Long-term oxygen therapy was administered to 11 (36.7\%) patients. Demographic characteristics of the study population are summarised in table 1 .

\section{Outcome (pulmonary function tests)}

The patients' mean \pm SD FVC \% predicted at treatment initiation (baseline) and at the end of 1, 2 and 3 years are shown in figure 2a. The annual rate of decline in FVC \% pred over the 3 -year treatment period 


\begin{tabular}{|c|c|}
\hline Patients & 30 \\
\hline Age years & $70.2 \pm 8.4$ \\
\hline \multicolumn{2}{|l|}{ Sex } \\
\hline Male & $26(86.7)$ \\
\hline Female & 4 (13.3) \\
\hline \multicolumn{2}{|l|}{ Smoking status } \\
\hline Ex-smokers & 19 (63.3) \\
\hline Never-smokers & $11(36.7)$ \\
\hline \multicolumn{2}{|l|}{ Antifibrotics } \\
\hline Pirfenidone & $14(46.7)$ \\
\hline Nintedanib & 16 (53.3) \\
\hline Identification of inciting antigen & $25^{\#}(83.3)$ \\
\hline Surgical lung biopsy & 7 (23.3) \\
\hline \multicolumn{2}{|l|}{ Comorbidities } \\
\hline GORD & $12(40)$ \\
\hline Pulmonary hypertension & $9(30)$ \\
\hline Arterial hypertension & $13(43.3)$ \\
\hline Diabetes & $6(20)$ \\
\hline Coronary artery disease & $6(20)$ \\
\hline Supplementary oxygen & $11(36.7)$ \\
\hline FVC $\%$ pred & $74.2 \pm 18.3$ \\
\hline$D_{\text {Lco }} \%$ pred & $46.9 \pm 16$ \\
\hline
\end{tabular}

adjusted for baseline FVC \% pred measurement was 4.2\% (95\% CI 1.9-6.6\%, p=0.001). The adjusted estimates of FVC \% pred at the end of 1,2 and 3 years are shown in table 2.

The aforementioned analysis was also performed after imputing the missing FVC \% pred data due to death to a value of $30 \%$ (figure $2 \mathrm{~b}$ ). The annual rate of decline in FVC \% pred over the 3-year treatment period adjusted for baseline FVC \% pred measurement was 7.5\% (95\% CI 3.3-11.7\%; $\mathrm{p}=0.001$ ). The baseline-adjusted estimates of FVC \% pred at the end of 1,2 and 3 years are shown in table 2 .

Regarding $D_{\text {LCO }}$ analysis, results are shown in figure $3 \mathrm{a}$ and $\mathrm{b}$ and table 2.
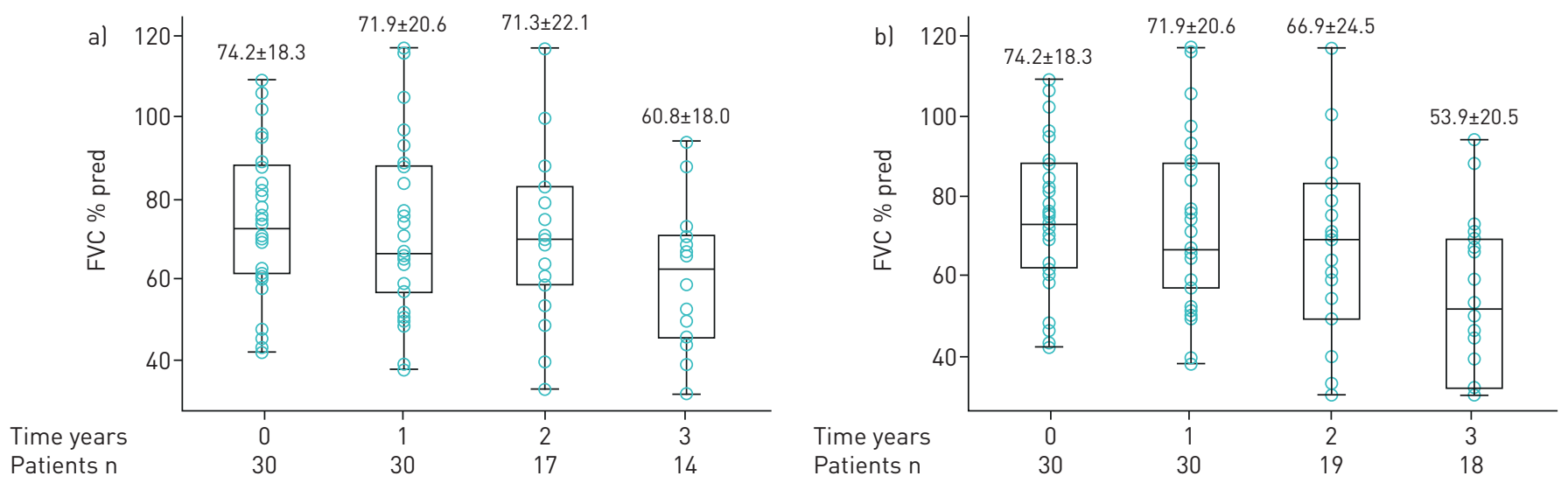

FIGURE 2 Mean \pm SD forced vital capacity (FVC) \% predicted values at 0 (baseline/treatment initiation), 1, 2 and 3 years following treatment with antifibrotics. a) Analysis based on as-observed (nonimputed) data. Data for all patients were available at 0 - and 1-year time points. At 2 years, we had available data for 17 patients, as two patients had died, and 11 patients had either been lost to follow-up or had not reached this time point. At 3 years, we had available data for 14 patients, as four patients had died and 12 patients had either been lost to follow-up or had not reached this time point. FVC \% pred values are normally distributed at all time points. b) Analysis after imputing measurements missing due to death. Missing FVC \% pred measurements for patients who died ( $n=2$ at 2 years and $n=4$ at 3 years) were imputed at a value of $30 \%$ pred, considered the lowest arbitrary value compatible with life. FVC \% pred values are normally distributed at all time points. 
TABLE 2 Estimates of the annual rate of decline in forced vital capacity (FVC) \% predicted and diffusing capacity of the lung for carbon monoxide $\left(D_{\mathrm{LCO}}\right) \%$ pred derived from linear mixed models based on the initial treatment assignment

\begin{tabular}{|c|c|c|c|c|c|}
\hline & Patients & Estimated annual rate of decline & \multicolumn{3}{|c|}{ Baseline-adjusted estimates } \\
\hline $\begin{array}{l}\text { FVC } \% \text { pred based on as-observed } \\
\text { (nonimputed) data }{ }^{\#}\end{array}$ & 30 & $\begin{array}{c}4.2 \\
(1.9-6.6) ; \\
p=0.001\end{array}$ & $\begin{array}{c}72.4 \\
(68.3-76.5)\end{array}$ & $\begin{array}{c}68.2 \\
(64.7-71.6)\end{array}$ & $\begin{array}{c}64.0 \\
(59.6-68.3)\end{array}$ \\
\hline FVC $\%$ pred including data imputed due to death" & 30 & $\begin{array}{c}7.5 \\
p=0.001\end{array}$ & $\begin{array}{c}72.0 \\
(68.1-75.9)\end{array}$ & $\begin{array}{c}64.5 \\
(59.4-69.7)\end{array}$ & $\begin{array}{c}57.0 \\
(48.3-65.7)\end{array}$ \\
\hline $\begin{array}{l}D_{\text {Lco }} \% \text { pred including data imputed } \\
\text { due to death }\end{array}$ & 24 & $\begin{array}{c}5.8 \\
(3.4-8.1) \\
p<0.001\end{array}$ & $\begin{array}{c}45.0 \\
(41.3-48.8)\end{array}$ & $\begin{array}{c}39.3 \\
(35.1-43.5)\end{array}$ & $\begin{array}{c}33.5 \\
(27.8-39.3)\end{array}$ \\
\hline
\end{tabular}

Data are presented as $\mathrm{n}$ or $\%(95 \% \mathrm{Cl})$, unless otherwise stated. ${ }^{\#}$ : linear mixed model using random intercept and slope with unstructured covariance matrix; ${ }^{\text {ๆ: }}$ linear mixed model using random intercept and slope with compound symmetry covariance matrix; six measurements ( $n=2$ at 2 years and $n=4$ at 3 years) were imputed to $30 \%$ due to death; ${ }^{+}$: linear mixed model using random intercept and slope with Toeplitz covariance matrix; ${ }^{\S}$ : linear mixed model using random intercept and slope with Toeplitz covariance matrix; $n=1$ measurement at 3 years was imputed to $15 \%$ due to death.

\begin{abstract}
Adverse events
Both pirfenidone and nintedanib exhibited an acceptable safety profile. The nature of adverse events was greatly related to the type of antifibrotics administered. The most common adverse event was diarrhoea, which was observed in nine $(30 \%)$ out of 30 patients, exclusively in those receiving nintedanib (nine (56.3\%) out of 16). Gastrointestinal upset was observed in four (13.3\%) patients: one (7.1\%) receiving pirfenidone and three (18.8\%) receiving nintedanib. Photosensitivity was observed in three (10\%) out of 30 patients, solely those receiving pirfenidone (three $(21.4 \%)$ out of 14 ). Fatigue was reported by three (10\%) out of 30 patients: one (7.1\%) out of 14 receiving pirfenidone and two (12.5\%) out of 16 receiving nintedanib. There were no cases of liver toxicity or bleeding. Adverse events were successfully managed in five (16.7\%) patients. In five (16.7\%) patients, a dose reduction was required to control adverse events,
\end{abstract}
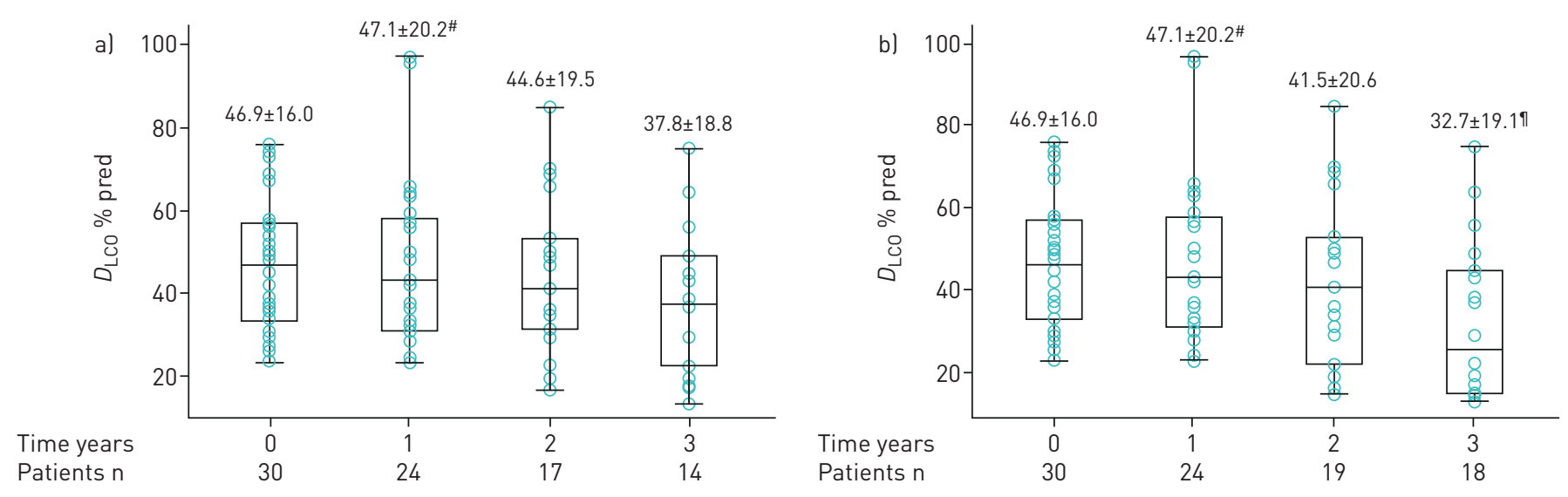

FIGURE 3 Mean \pm SD diffusing capacity of the lung for carbon monoxide $\left(D_{\mathrm{LCO}}\right) \%$ predicted values at 0 (baseline/treatment initiation), 1,2 and 3 years following treatment with antifibrotics. a) Analysis based on as-observed (nonimputed) data. Data for all patients were available at time 0 . At 1 year, data were available for 24 patients, as for six patients $D_{\text {Lco }}$ measurements were not available. At 2 years, we had available data for 17 patients, as two patients had died, and 11 patients had either been lost to follow-up or had not reached this time point. At 3 years, we had available data for 14 patients, as four patients had died, and 12 patients had either been lost to follow-up or had not reached this time point. $D_{\mathrm{Lco}}$ $\%$ pred values at 0,2 , and 3 years are normally distributed, while values at 1 year do not follow a normal distribution. b) Analysis after imputing measurements missing due to death. Missing data due to death ( $\mathrm{n}=2$ at 2 years; and $\mathrm{n}=4$ at 3 years) were imputed at a $D_{\mathrm{Lco}} \%$ predicted value of $15 \%$, considered the lowest arbitrary value compatible with life. $D_{1 \text { co }} \%$ predicted values at 0 and 2 years are normally distributed, while values at 1 and 3 years do not follow a normal distribution. ${ }^{\#}$ : median (interquartile range (IQR) 43.0 (31.0-58.0)\% pred; ${ }^{\text {ๆ }}$ : median (IQR) 25.5 (15.0-45.0)\% pred. 
while in another five (16.7\%) patients a permanent discontinuation of the drug was necessary. Most of the patients who underwent drug discontinuation switched successfully to the alternative antifibrotic agent (four (80\%) out of five). In one patient receiving nintedanib, permanent discontinuation was required due to diarrhoea. This patient did not consent to the initiation of pirfenidone. The incidence and management of adverse events are summarised in table 3 .

\section{Discussion}

This is an observational long-term study describing the clinical experience with antifibrotics in patients with f-HP diagnosed in the context of multidisciplinary team discussion over a period of 3 years. Both FVC $\%$ pred and $D_{\text {LCO }} \%$ pred exhibited a statistically significant annual decline over the course of our 3 year study. However, we must emphasise that the retrospective nature of the study and the absence of a comparator arm prohibits the drawing of any conclusions over efficacy. We did not perform subgroup statistical analysis between pirfenidone and nintedanib because of the small size of our cohort and also due to the fact that four patients switched to the alternative antifibrotic as a result of adverse events.

Both pirfenidone and nintedanib exhibited an acceptable safety profile. The frequency and type of adverse events are in line with those reported in the approval studies. Reflecting the difficulties in everyday clinical practice it is worth highlighting that $20 \%$ of patients discontinued therapy due to adverse events. The majority of these patients $(80 \%)$ were successfully switched to the alternative antifibrotic agent. All patients were referred to us within 1 month of treatment initiation. Thus, there were no cases of patients discontinuing treatment early on that could lead to under-reporting of adverse events.

Currently, available empirical treatment for f-HP is limited to the combination of immunomodulating agents based on small retrospective studies showing functional stabilisation with the use of low doses of corticosteroids and mycophenolate mofetil or azathioprine [20, 21]. SHIBATA et al. [22] performed a retrospective study and demonstrated that patients with f-HP $(n=23)$ exhibited significantly improved rates of decline at 6 months following treatment with pirfenidone compared to those 6 months prior to treatment initiation $(-292 \pm 77.8$ versus $152 \pm 56.1 \mathrm{~mL}, \mathrm{p}=0.047)$. In contrast to our study, none of the patients had to discontinue treatment due to drug-related side-effects, yet this was a short-term study and direct comparisons cannot be made. MATEOS-TolEDo et al. [23] demonstrated superiority of pirfenidone as add-on treatment to corticosteroids compared to azathioprine in quality-of-life parameters, but not in lung function tests, in a small open-label trial of 1 year duration, involving 22 patients with f-HP.

The classification of idiopathic interstitial pneumonias according to their clinical behaviour can vary from reversible and self-limited to progressive and irreversible despite therapy [15]. This scheme is applicable to other ILDs besides idiopathic cases, and f-HP represents a characteristic example given the wide variability of its pathology patterns and clinical behaviour. There are cases of f-HP with a relentless progression comparable to the inexorable progression of IPF [11].

Management for patients with f-HP is challenging. While immunosuppression has been used successfully in a number of patients [21], some patients exhibit a relentless progression. The clear unmet medical need

TABLE 3 Adverse events and their management recorded during the treatment period

\begin{tabular}{|c|c|c|c|}
\hline & Complete cohort & Pirfenidone & Nintedanib \\
\hline Type of adverse events & 30 & 14 & 16 \\
\hline Photosensitivity & $3(10)$ & $3(21.4)$ & $0(0)$ \\
\hline Diarrhoea & $7^{\#}(23.3)$ & $0(0)$ & $9^{\#}(56.3)$ \\
\hline Liver toxicity & $0(0)$ & $0(0)$ & $0(0)$ \\
\hline Bleeding & $0(0)$ & $0(0)$ & $0(0)$ \\
\hline Gastrointestinal upset & 4 (13.3) & $1(7.1)$ & $3(18.8)$ \\
\hline Fatigue & $3(10)$ & $1(7.1)$ & $2(12.5)$ \\
\hline Death & $5^{\text {ก }}(16.7)$ & 2 (14.3) & $3(18.8)$ \\
\hline \multicolumn{4}{|l|}{ Management of adverse events } \\
\hline Symptomatic management & $3(12)$ & $0(0)$ & $5(31.3)$ \\
\hline Dose reduction & $5(20)$ & $1(7.1)$ & $4^{\#}(25)$ \\
\hline Drug discontinuation & $5(20)$ & $3(21.4)$ & $2(12.5)$ \\
\hline Switch to alternative antifibrotic & $4(16)$ & $3(21.4)$ & $1(6.3)$ \\
\hline
\end{tabular}


to manage progressive fibrotic lung diseases led to the double-blind, placebo-controlled, INBUILD study [14]. This study included a heterogeneous population of 663 patients with progressive fibrotic lung disease. However, a significant number of patients with f-HP participated; specifically 173 patients, 84 in the nintedanib group and 89 in the placebo group. The study met its primary end-point, which was the annual rate of decline in FVC as assessed over a 52-week period. The benefit was similar to that observed in the approval studies of pirfenidone and nintedanib for IPF [24-26], irrespective of the underlying ILD diagnosis [27]. Subgroup analysis of the INBUILD study did not show any meaningful differences in the effect of nintedanib versus placebo across all five groups studied, including f-HP [27]. Our data suggest similar findings, although our study is smaller and not placebo controlled. In the subgroup analysis of the INBUILD study [27], in the f-HP group, diarrhoea was observed in $70 \%$, abdominal pain in $17 \%$ and an adverse event leading to permanent treatment discontinuation in $19 \%$ of patients, while in our study the respective rates were $56.3 \%, 18.8 \%$ and $12.5 \%$, respectively, in patients receiving nintedanib. In terms of adverse events, nintedanib exhibited a similar safety profile to that observed in the IPF clinical trials [28].

Similar therapeutic benefits were observed with pirfenidone in 256 patients with progressive fibrotic unclassifiable ILDs [29]. Finally, the SENSCIS trial of 576 patients with systemic sclerosis-associated ILD treated with nintedanib [30] demonstrated significant reductions in disease progression with no major safety concerns. More importantly, in all randomised controlled trials, treatment response rates were more pronounced in patients with a UIP-like phenotype, extending previous observations indicating similar prognosis in all UIP-like patterns, irrespective of disease aetiology [11]. These encouraging therapeutic data underline the necessity to lump progressive fibrotic ILDs on the basis of common disease behaviours and treatment responses [31].

We acknowledge limitations of our study. This is a retrospective, observational study of patients with f-HP receiving antifibrotics and it lacks a comparator arm. In addition, our study included a limited number of patients and therefore rigid conclusions on drug efficacy cannot be drawn. Additionally, considering that patients with IPF were newly diagnosed and treatment naïve, functional data prior to treatment initiation are unavailable.

Despite the limitations, our study has several strengths. It included exclusively well-characterised patients with $\mathrm{f}-\mathrm{HP}$ who were diagnosed in the context of multidisciplinary team discussion in two reference centres. Most notably, a significant number of patients were followed-up for a period of up to 3 years. This long period is useful as it provides more solid, clinically relevant information regarding the clinical course of patients with f-HP under antifibrotic treatment. In conclusion, both antifibrotics exhibited an acceptable safety profile in patients with f-HP. Despite the administration of antifibrotics, there was a statistically significant annual decline in FVC \% pred and $D_{\mathrm{LCO}} \%$ pred over a 3-year period. Long-term randomised controlled trials, preferably beyond 1 year, are needed.

Acknowledgements: The authors would like to thank Agoritsa Bismpiroula (Qualitis, Athens, Greece) for her assistance with the statistical analysis.

Conflict of interest: V. Tzilas has nothing to disclose. A. Tzouvelekis has nothing to disclose. E. Bouros has nothing to declare. T. Karampitsakos has nothing to disclose. M. Ntassiou has nothing to disclose. E. Avdoula has nothing to disclose. A. Trachalaki has nothing to disclose. K. Antoniou has nothing to disclose. G. Raghu reports consultancy for Roche, Genentech and Boerhinger Ingelheim. D. Bouros reports grants, personal fees and nonfinancial support from Boehringer Ingelheim and Roche, and grants from Elpen, outside the submitted work.

\section{References}

1 Walsh SLF, Wells AU, Desai SR, et al. Multicentre evaluation of multidisciplinary team meeting agreement on diagnosis in diffuse parenchymal lung disease: a case-cohort study. Lancet Respir Med 2016; 4: 557-565.

2 Selman M, Pardo A, King TE Jr. Hypersensitivity pneumonitis: insights in diagnosis and pathobiology. Am J Respir Crit Care Med 2012; 186: 314-324.

3 Vasakova M, Morell F, Walsh S, et al. Hypersensitivity pneumonitis: perspectives in diagnosis and management. Am J Respir Crit Care Med 2017; 196: 680-689.

4 Salisbury ML, Myers JL, Belloli EA, et al. Diagnosis and treatment of fibrotic hypersensitivity pneumonia. Where we stand and where we need to go. Am J Respir Crit Care Med 2017; 196: 690-699.

5 Vasakova M, Selman M, Morell F, et al. Hypersensitivity pneumonitis: current concepts of pathogenesis and potential targets for treatment. Am J Respir Crit Care Med 2019; 200: 301-308.

6 Barnes H, Morisset J, Molyneaux P, et al. A systematically derived exposure assessment instrument for chronic hypersensitivity pneumonitis. Chest 2020; 157: 1506-1512.

7 Morisset J, Johannson KA, Jones KD, et al. Identification of diagnostic criteria for chronic hypersensitivity pneumonitis: an international modified Delphi survey. Am J Respir Crit Care Med 2018; 197: 1036-1044.

8 Vourlekis JS, Schwarz MI, Cherniack RM, et al. The effect of pulmonary fibrosis on survival in patients with hypersensitivity pneumonitis. Am J Med 2004; 116: 662-668.

9 Hanak V, Golbin JM, Ryu JH. Causes and presenting features in 85 consecutive patients with hypersensitivity pneumonitis. Mayo Clin Proc 2007; 82: 812-816. 
10 Wang $\mathrm{P}$, Jones $\mathrm{KD}$, Urisman A, et al. Pathologic findings and prognosis in a large prospective cohort of chronic hypersensitivity pneumonitis. Chest 2017; 152: 502-509.

11 Salisbury ML, Gu T, Murray S, et al. Hypersensitivity pneumonitis: radiologic phenotypes are associated with distinct survival time and pulmonary function trajectory. Chest 2019; 155: 699-711.

12 Jinta T, Miyazaki Y, Kishi M, et al. The pathogenesis of chronic hypersensitivity pneumonitis in common with idiopathic pulmonary fibrosis: expression of apoptotic markers. Am J Clin Pathol 2010; 134: 613-620.

13 García de Alba C, Buendia-Roldán I, Salgado A, et al. Fibrocytes contribute to inflammation and fibrosis in chronic hypersensitivity pneumonitis through paracrine effects. Am J Respir Crit Care Med 2015; 191: 427-436.

14 Flaherty KR, Wells AU, Cottin V, et al. Nintedanib in progressive fibrosing interstitial lung diseases. $N$ Engl J Med 2019; 381: 1718-1727.

15 Travis WD, Costabel U, Hansell DM, et al. An official American Thoracic Society/European Respiratory Society statement: update of the international multidisciplinary classification of the idiopathic interstitial pneumonias. Am J Respir Crit Care Med 2013; 188: 733-748.

16 Morell F, Villar A, Montero MÁ, et al. Chronic hypersensitivity pneumonitis in patients diagnosed with idiopathic pulmonary fibrosis: a prospective case-cohort study. Lancet Respir Med 2013; 1: 685-694.

17 Ohshimo S, Bonella F, Cui A, et al. Significance of bronchoalveolar lavage for the diagnosis of idiopathic pulmonary fibrosis. Am J Respir Crit Care Med 2009; 179: 1043-1047.

18 Tzilas V, Tzouvelekis A, Bouros E, et al. Diagnostic value of BAL lymphocytosis in patients with indeterminate for usual interstitial pneumonia imaging pattern. Eur Respir J 2019; 54: 1901144.

19 Raghu G, Collard HR, Egan JJ, et al. An official ATS/ERS/JRS/ALAT statement: idiopathic pulmonary fibrosis: evidence-based guidelines for diagnosis and management. Am J Respir Crit Care Med 2011; 183: 788-824.

20 Adegunsoye A, Oldham JM, Fernández Pérez ER, et al. Outcomes of immunosuppressive therapy in chronic hypersensitivity pneumonitis. ERJ Open Res 2017; 3: 00016-2017.

21 Morisset J, Johannson KA, Vittinghoff E, et al. Use of mycophenolate mofetil or azathioprine for the management of chronic hypersensitivity pneumonitis. Chest 2017; 151: 619-625.

22 Shibata S, Furusawa H, Inase N. Pirfenidone in chronic hypersensitivity pneumonitis: a real-life experience. Sarcoidosis Vasc Diffuse Lung Dis 2018; 35: 139-142.

23 Mateos-Toledo H, Mejía-Ávila M, Rodríguez-Barreto Ó, et al. An open-label study with pirfenidone on chronic hypersensitivity pneumonitis. Arch Bronconeumol 2020; 56: 163-169.

24 Richeldi L, du Bois RM, Raghu G, et al. Efficacy and safety of nintedanib in idiopathic pulmonary fibrosis. $N$ Engl J Med 2014; 370: 2071-2082.

25 Noble PW, Albera C, Bradford WZ, et al. Pirfenidone in patients with idiopathic pulmonary fibrosis (CAPACITY): two randomised trials. Lancet 2011; 377: 1760-1769.

26 King TE Jr, Bradford WZ, Castro-Bernardini S, et al. A phase 3 trial of pirfenidone in patients with idiopathic pulmonary fibrosis. N Engl J Med 2014; 370: 2083-2092.

27 Wells AU, Flaherty KR, Brown KK, et al. Nintedanib in patients with progressive fibrosing interstitial lung diseases - subgroup analyses by interstitial lung disease diagnosis in the INBUILD trial: a randomised, double-blind, placebo-controlled, parallel-group trial. Lancet Respir Med 2020; 8: 453-460.

28 Lancaster L, Crestani B, Hernandez P, et al. Safety and survival data in patients with idiopathic pulmonary fibrosis treated with nintedanib: pooled data from six clinical trials. BMJ Open Respir Res 2019; 6: e000397.

29 Maher TM, Corte TJ, Fischer A, et al. Pirfenidone in patients with unclassifiable progressive fibrosing interstitial lung disease: design of a double-blind, randomised, placebo-controlled phase II trial. BMJ Open Respir Res 2018; 5 : e000289.

30 Distler O, Highland KB, Gahlemann M, et al. Nintedanib for systemic sclerosis-associated interstitial lung disease. N Engl J Med 2019; 380: 2518-2528.

31 Tzouvelekis A, Bouros D. Endotyping of progressive fibrotic interstitial lung diseases: it is the final destination that matters and not the journey. EBioMedicine 2020; 51: 102591. 